Nuclear Physics A436 (1985) 108-124

(C) North-Holland Publishing Company

\title{
PROPERTIES OF HOMOGENEOUS AND INHOMOGENEOUS MASS RELATIONS
}

\author{
J. JÄNECKE
}

Department of Physics, The University of Michigan, Ann Arbor, Michigan 48109, USA

and

E. COMAY

Department of Physics, The University of Michigan, Ann Arbor, Michigan 48109, USA and

Department of Physics and Astronomy, Tel-Aviv University, Ramat-Aviv, 69978, Tel-Aviv, Israel ${ }^{\dagger}$

Received 23 July 1984

\begin{abstract}
Mass equations based on third-order partial difference equations have been investigated. A test which makes use of subsets of data has been developed to study long-range extrapolations. Inherent connections with the liquid-drop model and the shell model are established. Higher-order effects in isospin, presumably due to subshell mixing and core polarization, are recognized as the origin for an inhomogeneous source term which strongly affects long-range extrapolations.
\end{abstract}

\section{Introduction}

The transverse Garvey-Kelson mass relation, which is a homogencous third-order partial difference equation, and its associated multi-parameter mass equation ${ }^{1,2}$ ) provide powerful tools for describing nuclear masses and binding energies. They have successfully been used for years to predict [e.g. refs. $\left.{ }^{1,3}\right)$ ] masses and binding energies of nuclei close to the known nuclei. However, new mass data for very proton-rich and very neutron-rich nuclei which have become available in recent years reveal difficulties with long-range extrapolations. Similar difficulties are observed for predictions based on the solutions of inhomogeneous third-order difference equations ${ }^{4}$ ) and of homogeneous fourth-order difference equations ${ }^{5,6}$ ). Furthermore, statistical tests could not convincingly establish the need for an inhomogeneous source term ${ }^{7,8}$ ).

A renewed attempt to study the underlying physical assumptions of mass equations using partial difference equations and their connections with other atomic mass models is called for. The availability of new mass data for nuclei far from the line of $\boldsymbol{\beta}$-stability suggested new systematic tests which have been used in this work to study and eventually explain the underlying reasons for the long-range characteristics.

+ Permanent address. 
Sect. 2 describes mass relations and the associated mass equations and their connection with the effective neutron-proton interaction. Sects. 3 and 4 discuss statistical tests and new procedures involving interpolations and extrapolations. In sects. 5 and 6 , finally, the shell dependence of the effective neutron-proton interaction and of the symmetry energy is established, including higher-order effects, and their influence on the solutions of inhomogeneous difference equations is studied.

\section{Mass relations, mass equations and the effective neutron-proton interaction}

Mass relations may be viewed as partial difference equations. Their solutions represent mass equations. Finding such solutions is a mathematical technique which usually leads to multi-parameter equations whereby, as is commonly done, the experimental data are used as initial or boundary condition.

The physical content of the procedure is contained in the structure of the difference equation. The physical content and not the mathematical technique determine the quality of an equation with regard to interpolations and extrapolations. For example, it was observed (see below) that the solutions of inhomogeneous third-order difference equations are extremely stable with regard to a large class of inhomogeneous source terms. This behavior is contrary to that observed for other multi-parameter procedures such as polynomial fitting.

The effective neutron-proton interaction $I_{\mathrm{np}}$ is defined by the operational equation ${ }^{7,9}$ )

$$
\begin{aligned}
I_{\mathrm{np}}(N, Z)= & B_{\mathrm{n}}(N, Z)+B_{\mathrm{p}}(N, Z)-B_{\mathrm{np}}(N, Z) \\
= & {[B(N, Z)-B(N-1, Z)]+[B(N, Z) \quad B(N, Z \quad 1)] } \\
& -[B(N, Z)-B(N-1, Z-1)] \\
= & B(N, Z)-B(N-1, Z)-B(N, Z-1)+B(N-1, Z-1) .
\end{aligned}
$$

Here, $B_{\mathrm{n}}, B_{\mathrm{p}}, B_{\mathrm{np}}$ and $B$ are the neutron, proton, neutron-proton and total binding energies. The transverse and longitudinal Garvey-Kelson relations follow directly from eq. (1) if it is assumed that $I_{\text {np }}$ is independent of isospin (neutron excess) or mass number, respectively. Simple shell-model considerations involving 4-fold degenerate Nilsson-like or Hartree-Fock single-particle levels or equations based on various seniority coupling schemes do indeed support the above assumptions.

The transverse and longitudinal homogeneous partial difference equations can be formulated as

$$
\begin{aligned}
& D_{\mathrm{T}} B(N, Z)=\Delta^{1,-1} \Delta^{1,0} \Delta^{0,1} B(N, Z)=0, \\
& D_{\mathrm{L}} B(N, Z) \equiv \Delta^{1,1} \Delta^{1,0} \Delta^{0,1} B(N, Z)=0,
\end{aligned}
$$

with partial difference operators

$$
\Delta^{i, j} f(N, Z)=f(N, Z)-f(N-i, Z-j) .
$$


The most general solutions of eqs. (2) and (3) are the well-known binding energy (or mass) equations

$$
\begin{aligned}
& B(N, Z)=G_{1}(N)+G_{2}(Z)+G_{3}(A), \\
& B(N, Z)=F_{1}(N)+F_{2}(Z)+F_{3}(E),
\end{aligned}
$$

with mass number $A=N+Z$ and neutron excess $E=N-Z$. Here, $G_{i}(k)$ and $F_{i}(k)$ are arbitrary point functions which are determined from least-squares adjustments to the data.

A homogeneous fourth-order partial difference equation $\left.{ }^{5,6}\right)$ is

$$
D_{\mathrm{TL}} B(N, Z) \equiv \Delta^{1,1} \Delta^{1,-1} \Delta^{1,0} \Delta^{0,1} B(N, Z)=0 .
$$

It has the solution

$$
B(N, Z)=H_{1}(N)+H_{2}(Z)+H_{3}(A)+H_{4}(E) .
$$

Eq. (7) assumes a particular smooth polynomial dependence of $I_{\text {np }}$ on $A$ and $E$.

More insight into the difference operators of eqs. (2)-(4) and (7) can be obtained when they are approximated by differential operators (neglecting pairing contributions). One recognizes the effective neutron-proton interaction $I_{\mathrm{np}}$ as a quantity related to the curvatures of the atomic mass surface (for $A=$ odd) with regard to $A$ and $E$,

$$
\begin{aligned}
I_{\mathrm{np}}(N, Z) & \equiv \Delta^{1,0} \Delta^{0,1} B(N, Z) \\
& \approx \frac{\partial^{2}}{\partial N \partial Z} B(N, Z)=\left(\frac{\partial^{2}}{\partial A^{2}}-\frac{\partial^{2}}{\partial E^{2}}\right) B(N, Z) .
\end{aligned}
$$

The other opcrators of eqs. (2), (3) and (7) represent derivatives of this curvature expression with respect to $E$ and/or $A$.

Inhomogeneous third-order partial difference equations are obtained from eqs. (2) and (3) by replacing the right-hand sides with non-vanishing inhomogeneous source terms $\tau_{\mathrm{T}}(N, Z)$ and $\tau_{\mathrm{L}}(N, Z)$. These must be derived from physical models. The solutions are similar to eqs. (5) and (6) except that a special solution of the inhomogeneous equation, usually symmetry and Coulomb energy terms, must be added on the right-hand sides. Various such equations have been discussed ${ }^{7}$ ) and predictions for one particular mass equation have been tabulated ${ }^{4}$ ). The adopted procedure of obtaining a special solution for an inhomogeneous difference equation is greatly simplified by directly constructing analytical expressions for the special solution rather than the source terms $\tau_{\mathrm{T}}(N, Z)$ and $\tau_{\mathrm{L}}(N, Z)$. The latter can subsequently be obtained by applying the operators $D_{\mathrm{T}}$ and $D_{\mathrm{L}}$ of eqs. (2) and (3).

Evidence for the possible existence of inhomogeneous source terms is so far essentially only of an indirect nature. The transverse and longitudinal Garvey-Kelson relations are based on identical physical models. Nevertheless, the mass eqs. (5) and (6) lead to diverging predictions ${ }^{9}$ ) away from the line of $\beta$-stability. This may suggest the influence of an inhomogeneous term. 
Finite inhomogeneous contributions are also suggested by mass models which contain an $\boldsymbol{A}$-dependent symmetry energy term. Calculating the effective neutronproton interaction $I_{\mathrm{np}}$ of eq. (1) from the liquid-drop model equation (for example) yields an expression ${ }^{7}$ ) which depends on mass number $A$ as well as isospin $T$ [see also ref. $\left.{ }^{10}\right)$ ]. The inhomogeneous source terms $\tau_{\mathrm{T}}(N, Z)$ and $\tau_{\mathrm{L}}(N, Z)$ can thus be estimated and are found to be very small but finite. For $A>40$ the most important contributions are $<10 \mathrm{keV}$ (transverse) and $<50 \mathrm{keV}$ (longitudinal) in magnitude and result from the symmetry energy term. Even smaller contributions of at most 1.0 to $1.5 \mathrm{keV}$ come from other terms. The question arises, of course, whether such small contributions are significant in the application of the homogeneous equations.

\section{Statistical tests}

Eqs. (2) and (3) are well satisfied by the experimental data. Using all possible mass combinations with $T_{z}>0$ and $N=Z=$ even from a recent mass evaluation ${ }^{11}$ ), mean and standard deviation of the relation inaccuracies are

$$
\begin{array}{ll}
R_{\mathrm{T}}=+15 \pm 222 \mathrm{keV} & (809 \text { cases }), \\
R_{\mathrm{L}}=-13 \pm 235 \mathrm{keV} & \text { (935 cases) }
\end{array}
$$

The relations are better satisfied for heavier nuclei than for the lighter ones as indicated by the standard deviations,

$$
\begin{gathered}
R_{\mathrm{T}}=\left\{\begin{aligned}
26 \pm 348 \mathrm{keV} & (157 \text { cases for } A=4 \text { to } 80) \\
9 \pm 193 \mathrm{keV} & (397 \text { cases for } A=81 \text { to } 160) \\
17 \pm 144 \mathrm{keV} & (236 \text { cases for } A=161 \text { to } 255),
\end{aligned}\right. \\
R_{\mathrm{L}}=\left\{\begin{aligned}
-40 \pm 367 \mathrm{keV} & (193 \text { cases for } A=4 \text { to } 80) \\
-7 \pm 208 \mathrm{keV} & (423 \text { cases for } A=81 \text { to } 160) \\
-5 \pm 134 \mathrm{keV} & (278 \text { cases for } A=161 \text { to } 255) .
\end{aligned}\right.
\end{gathered}
$$

Also, the relations appear to be slightly better for proton-rich than for neutron-rich nuclei.

Both the transverse and the longitudinal relations are essentially equally well satisfied. The mean values may reflect upon systematic inhomogeneous contributions. However, they are significantly smaller than the random fluctuations. Eqs. (10) and (11) establish the expected accuracy of the homogeneous mass predictions based on these mass relations.

Random prediction errors expected in the repeated application of the relations should be equal to those for the mass eqs. (5) and (6). These effects have bcen studied by Comay and Kelson ${ }^{12}$ ) and were found to increase for the transverse equation with increasing distance $D$ from the line of $\beta$-stability approximately as 
$\sigma \simeq(42 \mathrm{keV}) D^{3 / 2}$. Systematic prediction errors from constant residuals should not exceed the random errors for $D \leqslant 11$ [ref. $\left.\left.{ }^{12}\right)\right]$. Small anomalous correlations are observed in another recent statistical study ${ }^{13}$ ), but a randomness assumption which connects the standard deviations of the residuals of eq. (10) to those for the mass equations (5) and (6) appears to be globally satisfied.

Since it has been recognized ${ }^{7}$ ) that the effective interaction $I_{n p}$ of eq. (1) plays a key role in the understanding of the homogeneous and inhomogeneous mass relations, experimental differences $\Delta^{i j} I_{\text {np }}(i+j=$ even $)$ for $(i, j)=(1,1),(2,2)$, $(1,-1),(2,-2),(2,0)$ and $(0,2)$ have been studied to establish a dependence on $A, T, N$ and/or $Z$ and compared to model predictions. This earlier statistical analysis ${ }^{7}$ ) was performed separately for different classes of all nuclei, and the results suggested a weak dependence on $T$ and $A$ but with rather low statistical significance. In a similar global analysis ${ }^{5}$ ) experimental differences $\Delta^{i, j} I_{\mathrm{np}}$ with even larger values of $i$ and $j$ were found to be very small and compatible with zero.

It is concluded that statistical tests provide only weak evidence, if any, for the existence of an inhomogeneous term.

\section{Interpolations and extrapolations}

The most direct and only truly convincing test of a mass equation consists of the comparison of extrapolations with mass data not included in the determination of the mass parameters. Usually, as in the 1975 mass predictions, all available data are used in the determination of the parameters. The standard deviations are determined, and the predictive power of extrapolations is only tested as new data are measured over the years.

A more satisfactory approach is taken in the present work. All available data are systematically divided into subsets. Only one particular subset is used to determine the mass parameters, and all remaining data are subsequently compared to the predictions.

Fig. 1 shows one example. Here, the differences between experimental ${ }^{11}$ ) and calculated masses are displayed as a function of neutron and proton numbers. The calculated values are from eq. (5) which is the solution of the homogeneous transverse third-order partial difference eq. (2). Only data for a band of nuclei along the line of $\beta$-stability are used as input. The condition is $E_{\text {stab }}-D<E \leqslant E_{\text {stab }}+D$ with $E=N-Z$ and $E_{\text {stab }}=0.4 A^{2} /(200+A)$. The width in fig. 1 was taken as $W=2 D=6$. The data in this band (upper part of fig. 1) are extremely well reproduced with a standard deviation of $55 \mathrm{keV}$, and the maximum residuals are $-280 \mathrm{keV}$ and $+187 \mathrm{keV}$, respectively. The differences for the extrapolated $\mathrm{n}$-rich and p-rich nuclei are displayed in the lower part of fig. 1. Strong systematic departures of up to $+4 \mathrm{MeV}$ for the $\mathrm{n}$-rich subset and $-7 \mathrm{MeV}$ for the p-rich subset are apparent. These residuals exhibit shell behavior, and they are mostly positive and negative for the respective $\mathrm{n}$-rich and $\mathrm{p}$-rich subsets. 

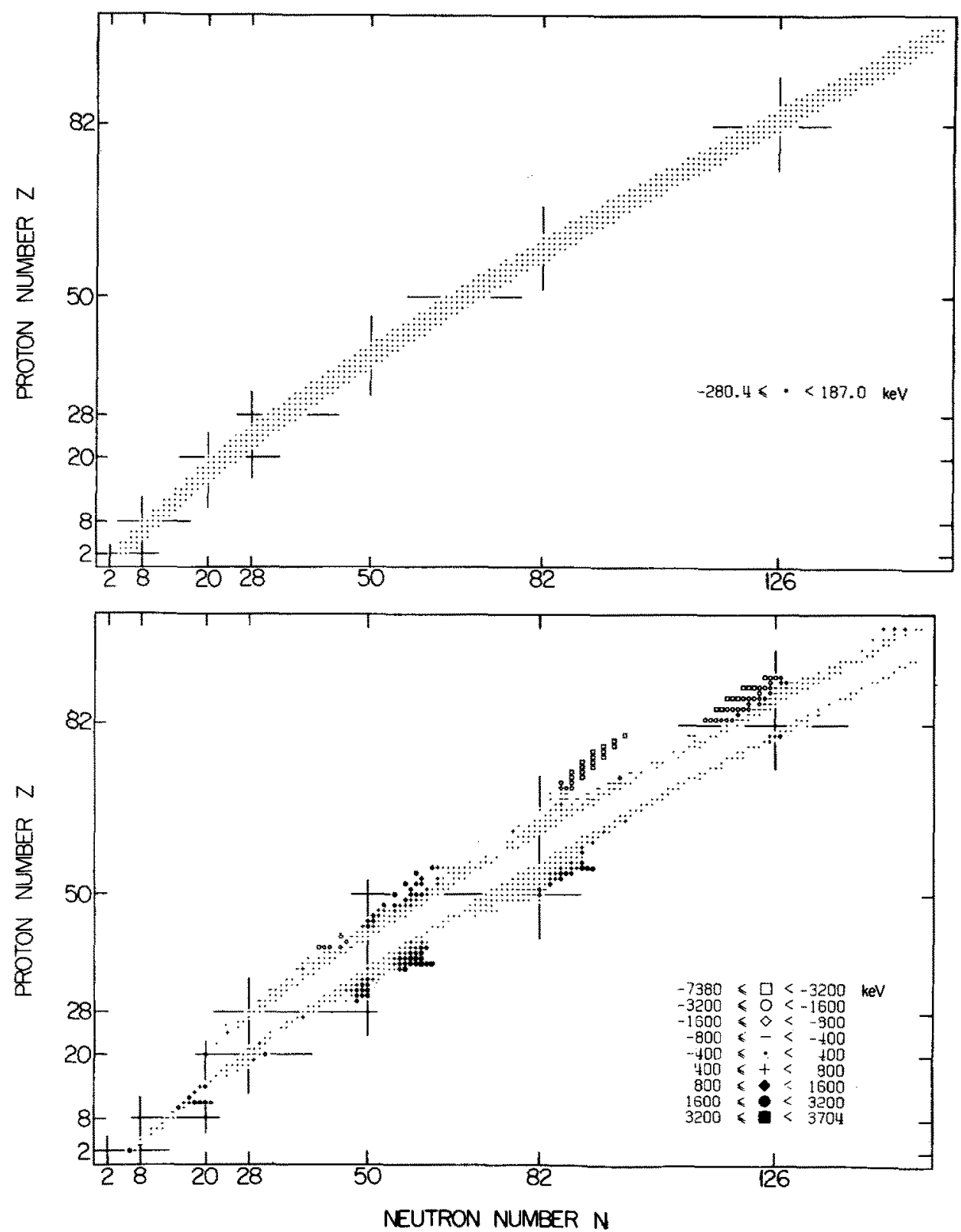

Fig. 1. Differences between experimental and calculated masses from the transverse mass eq. (5). The symbols denote nuclei with differences in the indicated ranges. Top: subset of nuclei used to determine the parameters. Bottom: subsets of extrapolated nuclei not used in the fitting procedure (see text). 


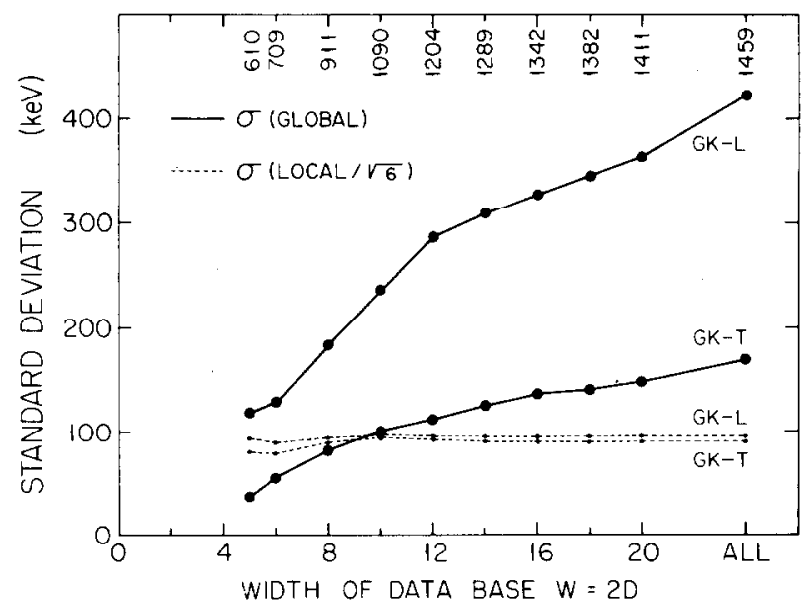

Fig. 2. Standard deviations for the differences between experimental and calculated masses (solid lines) and for the residuals of the mass relations divided by $\sqrt{6}$ (dashed lines) as function of the width $W=2 D$ of the assumed data base along the line of $\beta$-stability. Two curves each are shown for the transverse and longitudinal equations. The number of nuclei in the data base used for the calculations is given on top.

Similar results and graphs (not shown) have been obtained by using bands of different width $W$ as data base for the determination of the parameters.

The following discussion will first concentrate on the results for the nuclei along the stability line (data base) and then on the extrapolations.

Fig. 2 displays the standard deviations for reproducing the input mass data as function of the band width $W$. Results are shown for both, the transverse and the longitudinal equation. The standard deviations for the former increases slowly from $<100 \mathrm{keV}$ to $170 \mathrm{keV}$, for the latter from $\sim 100 \mathrm{keV}$ to $>400 \mathrm{keV}$. The superiority of the transverse equation is evident. The increase with $W$ is stronger than expected from the increase in the number of data $\left(\chi^{2}\right.$ per degree of freedom $f=n-p$ increases by a factor $\sim 4$ for the transverse and even more for the longitudinal equation). This increase with $W$ suggests systematic effects.

Also included in fig. 2 (dashed lines) are the standard deviations for the residuals of the transverse and longitudinal relations (similar to eq. (10)) divided by $\sqrt{6}$ as function of band width $W$. These residuals are independent of $W$ and they are essentially equal for the transverse and longitudinal relations. The factor $\sqrt{6}$ has been introduced to determine the validity of the randomness criterion of Comay and Kelson ${ }^{13}$ ) which requires $\sigma$ (prediction errors) $\approx \sigma$ (relation inaccuracies $) / \sqrt{6}$. This necessary condition for the standard deviations is not well satisfied for $D=$ $\frac{1}{2} W>6$ (transverse) and $D=\frac{1}{2} W>2$ (longitudinal). Anomalous correlations between the relation inaccuracies have already been observed ${ }^{13}$ ) and the above result is therefore not too surprising.

The increased difficulty of reproducing the experimental data with increasing bandwidth $W$ is also apparent from fig. 3. Here, the most positive and most negative 


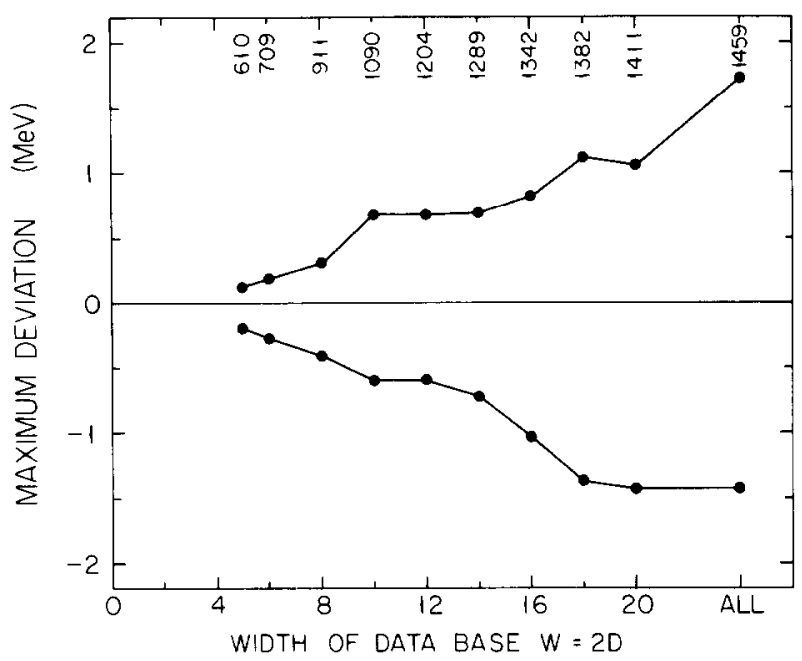

Fig. 3. Maximum positive and negative differences between experimental and calculated masses (within the data base) from the transverse mass equation as function of the width $W=2 D$ of the assumed data base. The number of nuclei in the data base used for the calculations is given on top.

deviations are plotted for the input data and are found to increase with $W$ even more strongly than the standard deviations.

The systematic errors of extrapolations based on the transverse and longitudinal equations are apparent from fig. 4. The mean differences between the experimental and the extrapolated masses are plotted as function of their distance $D$ from the line of $\beta$-stability for narrow bands of nuclei with $D=4$ to 6,6 to 8 and so on for n-rich nuclei and similarly for $\mathrm{p}$-rich nuclei.

Results for five calculations are shown with data bases of width $W=4,8,12,16$ and 20. Strong systematic errors are apparent. The predictions for both equations deviate in the same direction, and the discrepancies for the longitudinal equation are more pronounced (except $D<-14$ ). Proton-rich nuclei are on the average significantly more stable than predicted (by several $\mathrm{MeV}$ ), and neutron-rich nuclei are less stable. For data bases of small width, short-range extrapolations are quite good (transverse only). However, for data bases with large width the systematic effects become apparent already inside the region of nuclei used as data base.

Sample calculations similar to the ones just described for the solutions of the homogeneous third-order partial difference equations have also been performed for inhomogeneous third-order equations ${ }^{4,8,14}$ ) and the homogeneous fourth-order equations ${ }^{5,6}$ ). Essentially the same kind of systematic effects were observed. Thus, smooth inhomogeneous liquid-drop model Coulomb and symmetry energy terms appear to have little influence on long-range extrapolations.

Fig. 4 suggests that a phenomenological correction term of the type const $(T-$ $\left.T_{\text {stab }}\right)^{3}$ might reduce the systematic effects. However, the mean differences on 

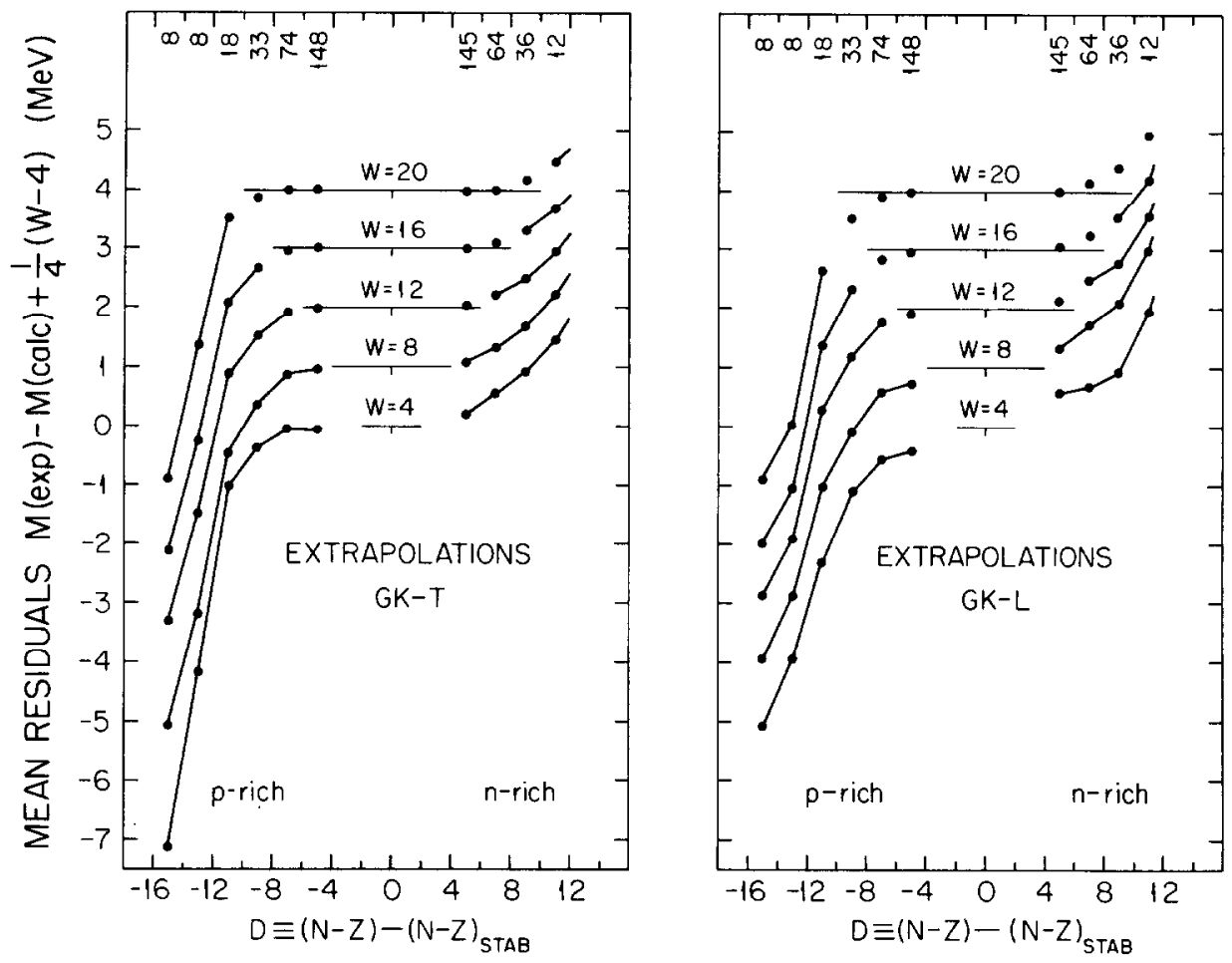

Fig. 4. Extrapolated mean values for the differences between experimental and calculated masses. The horizontal bars (displaced upward by $1 \mathrm{MeV}$ for each case) represent the increasing width of the data base. The calculations are based on the transverse (left) and longitudinal (right) eqs. (5) and (6), respectively. The number of extrapolated mass values is indicated at the top of the figure.

fig. 4 represent only a general trend, and the fluctuations of the individual differences about the mean values are typically of the same order of magnitude as the mean deviations. This property is directly apparent from the 2-dimensional display of fig. 1 , and the above procedure is not recommended.

The present chapter shows that a study of extrapolations based on a systematic variation of the data base and accompanying reevaluation of the mass parameters provides a very powerful method of testing the goodness of a mass equation.

\section{Shell-dependent neutron-proton interactions and symmetry energies}

Attempts to understand the observed systematic effects may take advantage of the inherent connections between the difference eqs. (2) and (3) and the effective interaction $I_{n p}$ and the symmetry energy. Experimental values of $I_{n p}$ according to eq. (1) have been obtained earlier [fig. 3 of ref. $\left.{ }^{7}\right)$, and it was observed that the splitting for even- $A$ and odd- $A$ nuclei $\left(I_{\mathrm{np}}(\operatorname{even}-A)>I_{\text {np }}(\right.$ odd $\left.-A)\right)$ as well as the general decrease with increasing mass number $A$ are quite well described by simple 
liquid-drop model equations. It was this fact, of course, which led to the inhomogeneous source terms introduced earlier ${ }^{4,8}$ ).

In an attempt to deduce additional information, a two-dimensional plot similar to fig. 1 of $I_{\text {np }}(\exp )-I_{\text {np }}$ (calc;LDM) as a function of $N$ and $Z$ (not shown) was constructed but showed essentially only random fluctuations. However, systematic shell effects became immediately apparent when the related quantities $I_{0}$ and $I^{\prime}$ of eq. (12) were displayed in a similar way.

One of the earliest investigations of the effective neutron-proton interaction is that of de-Shalit ${ }^{15}$ ) who observed that $I_{\mathrm{np}}$ can be written as

$$
I_{\mathrm{np}}=I_{0}+(-1)^{\mathrm{A}} I^{\prime} \text {. }
$$

Here, $I_{0}$ represents the spin-averaged neutron-proton interactions, and $I^{\prime}$ accounts for the increased binding in the ground state of an odd-odd nucleus where the spins $j_{\mathrm{n}}$ and $j_{\mathrm{p}}$ are coupled to the particular $J_{\mathrm{G} . S}$. The quantity $I^{\prime}$ accounts for the different splittings between the even-even and odd- $\boldsymbol{A}$ and the odd-odd and odd- $\boldsymbol{A}$ mass surfaces.
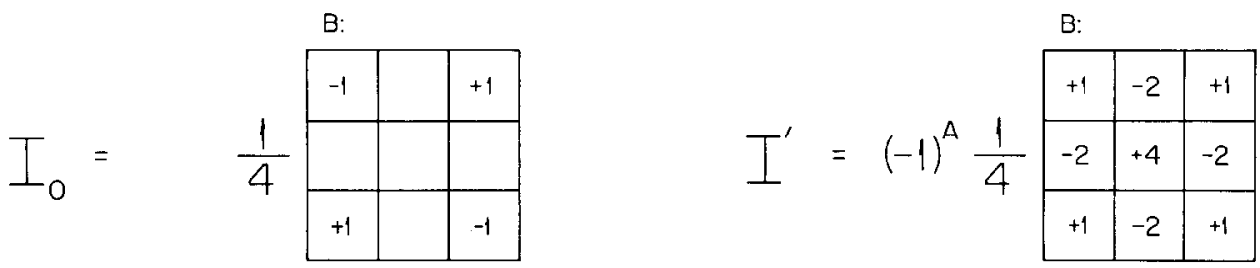

Fig. 5. Schematic representation of the two contributions to the effective neutron-proton interaction $I_{\mathrm{np}}=I_{0}+(-1)^{A} I^{\prime}$. Here, $I_{0}$ is the $J$-averaged interaction and $I^{\prime}$ is the enhancement for the ground states of odd-odd nuclei with $J=j_{\mathrm{n}}+j_{\mathrm{p}}$.

Calculating sums and differences of $I_{\mathrm{np}}$ according to eqs. (1) and (12) over four neighboring nuclei leads to simple expressions for $I_{0}$ and $I^{\prime}$. They are displayed schematically in fig. 5. Particularly $I_{0}$ (averaged over four neighboring nuclei) has a very simple structure. The quantity $I_{0}(\exp )-I_{0}($ calc;LDM) of fig. 6 calculated from this expression and recent mass data ${ }^{11}$ ) displays significant systematic departures from the smooth liquid-drop model predictions. This is particularly apparent at the shell crossings near ${ }^{208} \mathrm{~Pb}$ where $I_{0}>I_{0}(\mathrm{LDM})$ holds for the regions where neutrons and protons occupy orbits in different major shell regions ("NE" and "SW" of ${ }^{208} \mathrm{~Pb}$ ) whereas $I_{0}<I_{0}(\mathrm{LDM})$ holds for the "diagonal" region $82 \leqslant N, Z \leqslant$ 126 (114) ("NW" of ${ }^{208} \mathrm{~Pb}$ ). Equally pronounced effects are observed (not shown) for the quantity $I^{\prime}(\exp )-I$ (calc;LDM).

A shell dependence of $I_{0}$ and $I^{\prime}$ requires a corresponding shell dependence of the symmetry energy. Shell effects in $E_{\text {sym }}$ can indeed be observed by considering Coulomb-energy-corrected binding energies $B^{*}=B(\exp )+E_{\text {Coul }}($ calc $)$. Recent mass data ${ }^{11}$ ) have been used for the experimental binding energies. The total Coulomb energies in this equation have been calculated from recent shell-model 


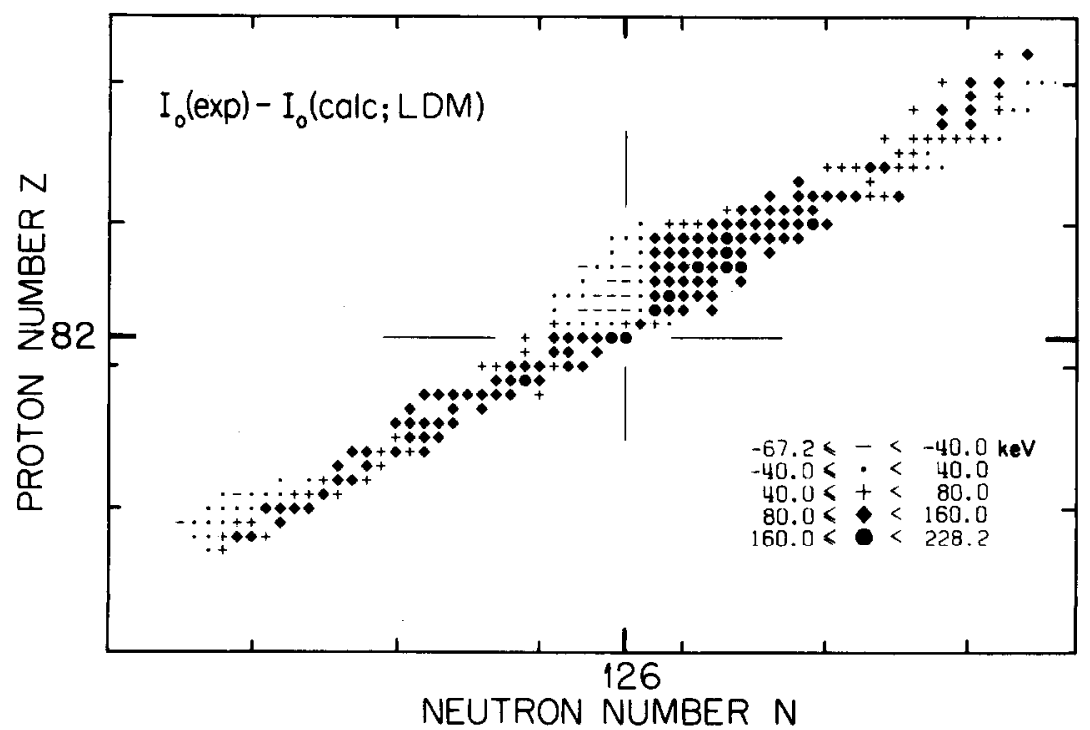

Fig. 6. $J$-averaged neutron-proton interaction $I_{0}(\exp )-I_{0}$ (calc; LDM) for nuclei from $A=150$ to 250 as function of $N$ and $Z$. The various symbols denote nuclei with positive or negative residuals in the indicated energy ranges.

expressions ${ }^{16,17}$ ) which express total Coulomb energies, Coulomb displacement energies and isoscalar, isovector, and isotensor Coulomb energies for nuclei with protons and neutrons in the same and in different major shell regions. All experimental Coulomb displacement energies are described with a standard deviation of 30 to $40 \mathrm{keV}$. The Coulomb-energy-corrected binding energies $B^{*}$ are displayed in fig. 7 for odd- $A$ nuclei as function of $T(T+1) / A$. Values for nuclei with the same value of $A$ are connected by straight lines. In the simplest liquid-drop model these lines should be parallel and the spacing should change continuously from light to heavy nuclei. This is approximately the case, but a closer inspection displays striking changes in slope at shell crossings (marked by dashed and dotted lines). In the off-diagonal shell regions where neutrons and protons are in different major shells, the binding energies decrease more strongly with isospin than in the diagonal shell regions. The slopes $a(A)$ in the symmetry energy expression $E_{\text {sym }}=$ $(a(A) / A) T(T+1)$ are plotted in fig. 8 separately for the diagonal and off-diagonal shell regions. The coefficients $a(A)$ are approximately constant for the heavier nuclei as expected from the simple liquid-drop model. Most importantly, the coefficients $a(A)$ for the off-diagonal regions are typically $15 \%$ bigger than for the diagonal regions. The shell dependence of the symmetry energy and the effective np interaction is related to properties of effective $T=0$ and $T=1$ nucleon-nucleon interaction energies for nucleons in the same or different major shell regions ${ }^{18}$ ).

The preceding considerations show that the properties of nuclear binding energies require an inhomogeneous source term in eq. (2) (as well as eq. (3) which will not 


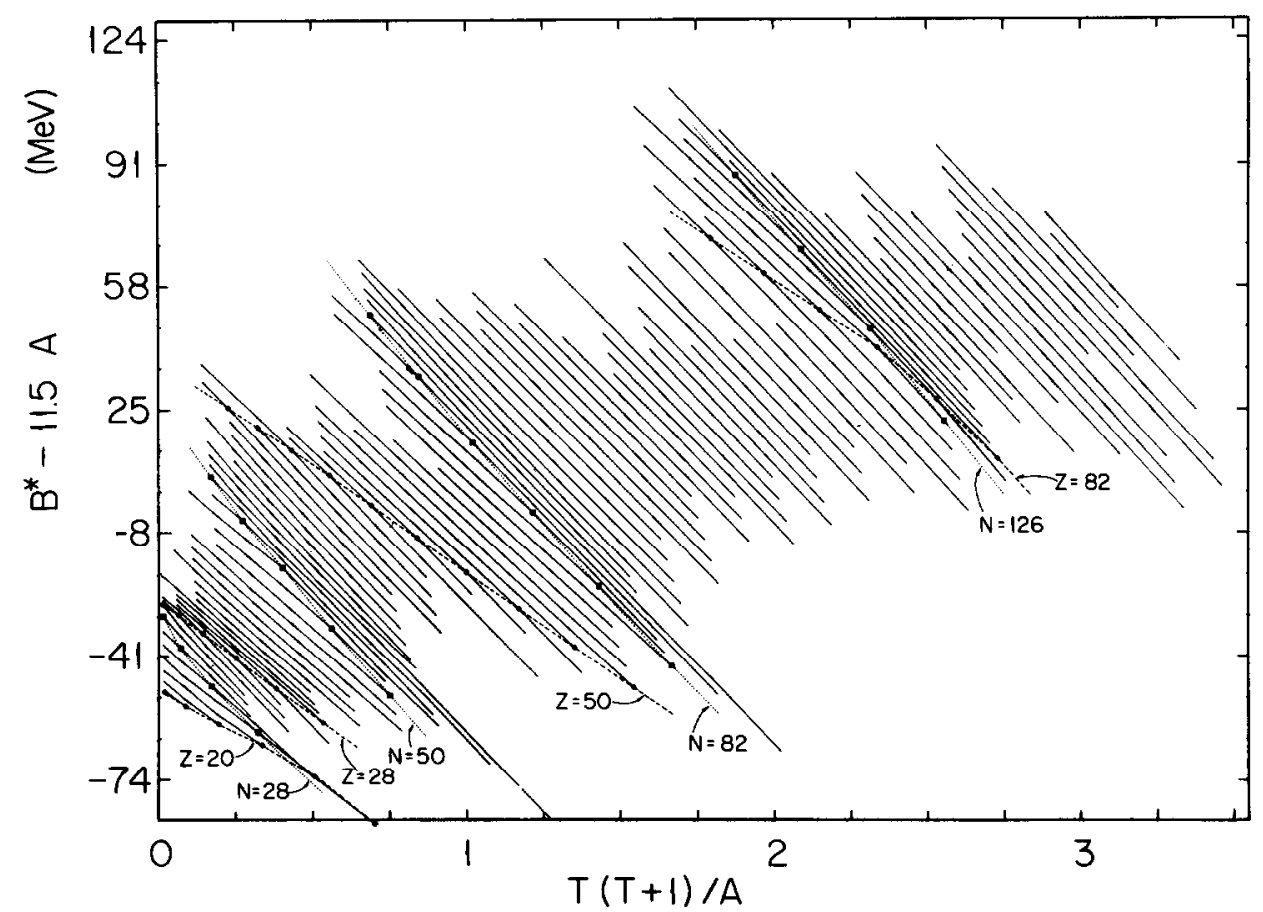

Fig. 7. Coulomb-energy-corrected binding energies $B^{*}=B(\exp )+E_{\text {Coul }}$ (calc) as function of $T(T+1) / A$ for odd- $A$ nuclei. Nuclei for the same values of $A$ (shifted vertically) are connected by solid lines. Semi-magic nuclei are connected by dashed and dotted lines.

be considered any longer) due to the shell-dependence of the symmetry energy. Using the techniques mentioned in sect. 2, a solution of the inhomogeneous thirdorder partial difference equation has been obtained. It explicitly includes shell-model Coulomb energy ${ }^{16,17}$ ) and shell-model symmetry energy contributions. The latter were constructed ${ }^{18}$ ) with a minimal number of three parameters per shell region (strength and size factors) to satisfy the overall characteristics of the symmetry energy of fig. 7.

Tests identical to those described in sect. 4 were performed for this new mass equation. Fig. 9 displays the extrapolations obtained with a data base of width $W-6$ corresponding to the lower part of fig. 1. Comparison between figs. 9 and 1 shows the unexpected and startling result that the inclusion of the rather detailed shell-model Coulomb energy and symmetry energy terms leads to only minor improvements in long-range extrapolations. The solutions of the difference equation display a remarkable degree of stability with respect to the added inhomogeneous terms. Only extrapolations in the region " $\mathrm{NW}$ " of ${ }^{208} \mathrm{~Pb}$ are improved, and the extreme positive and negative differences of the extrapolations are reduced by a mere $5 \%$. 

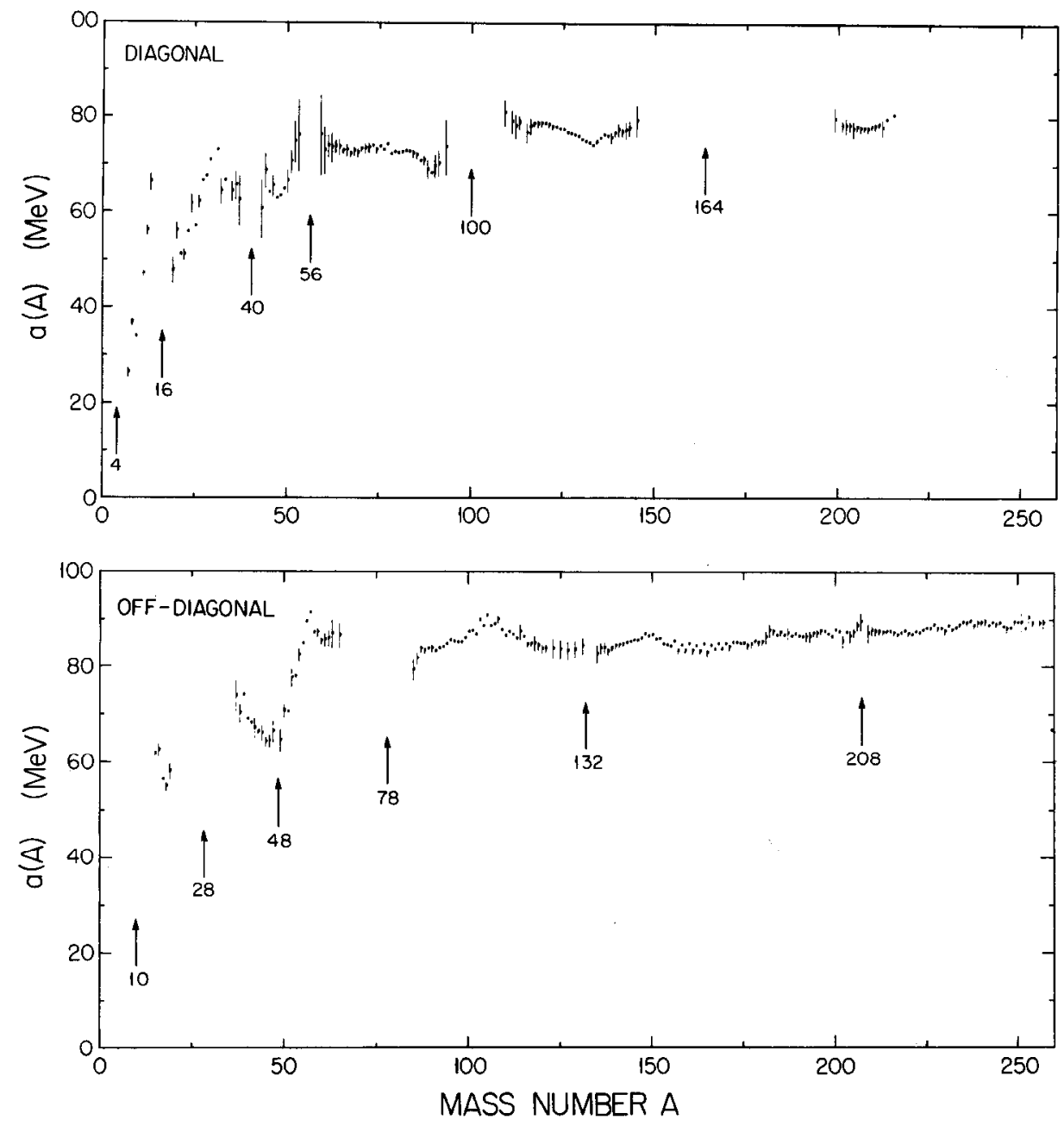

Fig. 8. Symmetry energy coefficient $a(A)$ in the expression $E_{\text {sym }}=(a(A) / A) T(T+1)$ as function of $A$ for diagonal and off-diagonal regions (neutrons and protons in same or different major shells).

The above result explains why the earlier mass predictions using third-order inhomogeneous ${ }^{4}$ ) and fourth-order homogeneous ${ }^{5}$ ) equations did not improve on the homogeneous third-order ${ }^{3}$ ) predictions.

\section{Higher-order effects in isospin}

The results of the preceding section suggest that higher-order effects in isospin may play a significant role for long-range extrapolations. This suggestion is supported by fig. 4 which points to cubic contributions. 


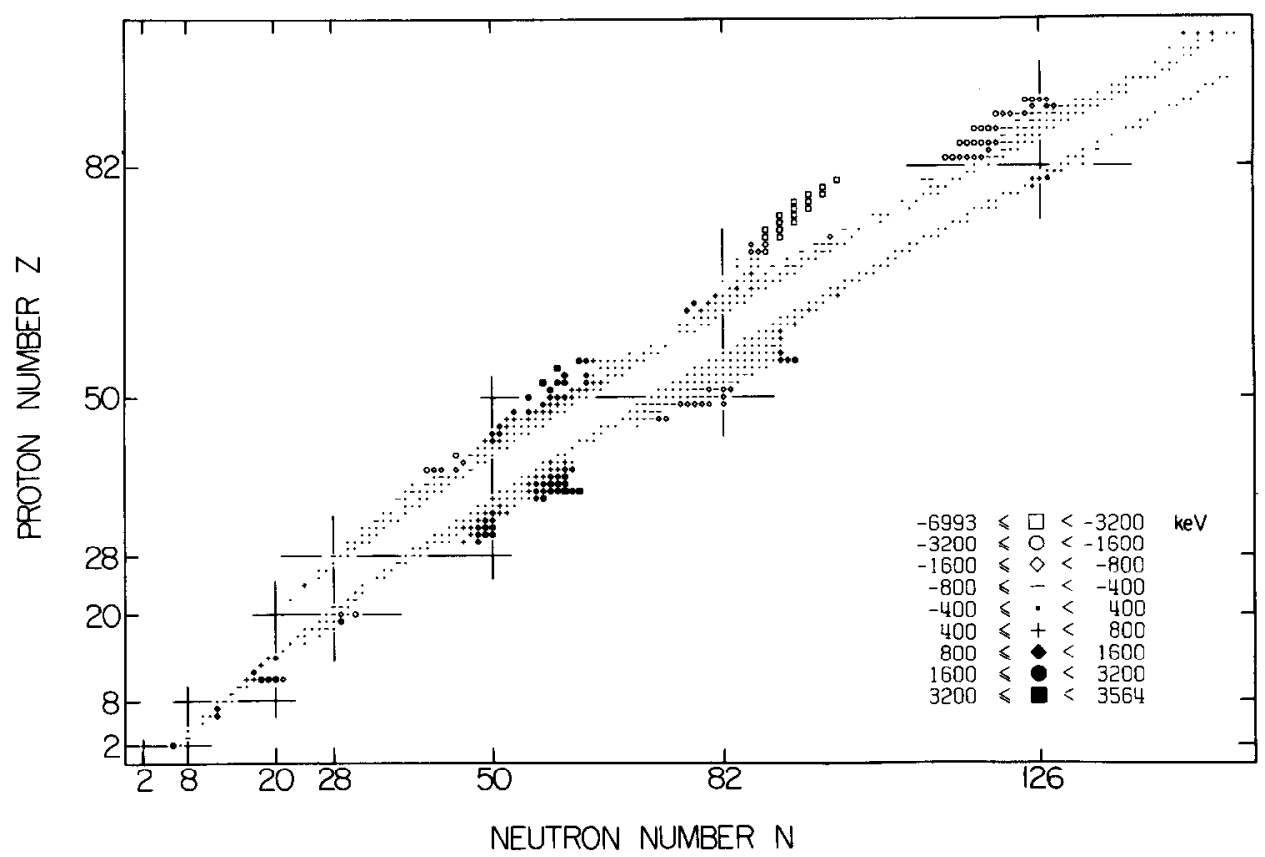

Fig. 9. Diflerences between experimental and calculated (extrapolated) masses from the transverse mass equation with inhomogeneous shell-dependent Coulomb energy and symmetry energy terms. (Compare to the lower part in fig. 1.)

A local Taylor expansion of the atomic mass surface as function of $n=N-N_{0}$ and $z=Z-Z_{0}$ (without pairing) for a nucleus $\left(N_{0}, Z_{0}\right)$ contains terms with $n, z, n^{2}$, $n z, z^{2}$ and so on. These terms are schematically arranged in fig. 10. The solutions of the homogeneous transverse mass equation includes all terms $n^{i}$ and $z^{i}$ but also the term $n z$ because of $2 n z=a^{2}-n^{2}-z^{2}$ with $a=A-A_{0}$. These terms are enclosed between the solid lines. Certain combinations of the other terms are also included in the solutions because of $a^{i}=(n+z)^{i}$. It is easy to show that the leading correction term not included in the solutions is proportional to $E^{3}$ (or $A^{2} E$ ) with $E=N-Z$.

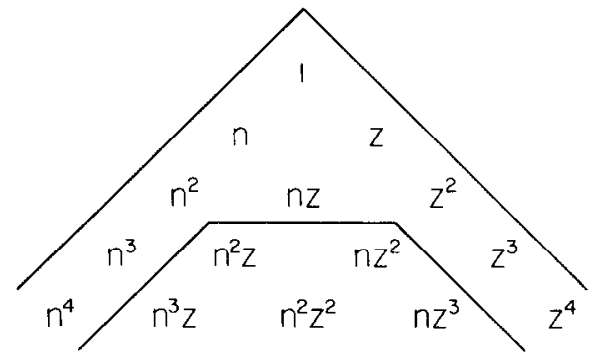

Fig. 10. Systematic arrangement of the expansion terms in a local Taylor expansion of the nuclear mass surface (without pairing). Only the terms between the solid lines are included in solutions of the homogeneous third-order partial difference equations. 
The leading correction terms for the longitudinal equation and for the fourth-order equation are obtained accordingly.

Contributions which are cubic in neutron excess or isospin enter a mass equation only as higher-order isospin correction terms. Writing the Coulomb-energy-corrected binding energy as

$$
B^{*}(N, Z)=f(A)-\left(\alpha_{1} T+\alpha_{2} T^{2}+\alpha_{3} T^{3}\right)
$$

yields

$$
\begin{gathered}
I_{\mathrm{np}}(N, Z)=(f(A)-2 f(A-1)+f(A-2))+\frac{1}{2} \alpha_{2}+\frac{3}{2} \alpha_{3} T, \\
\tau_{T}(N, Z)=-\frac{3}{2} \alpha_{3} .
\end{gathered}
$$

Thus, a cubic dependence on isospin leads to a constant inhomogeneous source term in the transverse partial difference equation.

Testing the long-range extrapolations with the procedure of sect. 4 for the equation which contains shell-dependent Coulomb and symmetry energy terms and added cubic isospin terms shows extreme sensitivity to these added terms. It also became apparent that the cubic contributions must be shell-dependent, and furthermore that a weak dependence on $A$ exists within each shell region occasionally requiring a change in sign. Introducing a phenomenological expression $p(A)\left(T-T_{\text {stab }}\right)^{3}$ into the special solution of the inhomogeneous mass equation leads to a remarkable improvement in long-range extrapolations as evidenced by fig. 11 . Here, extrapolations for the off-diagonal shell region $N=82$ to $126, Z=50$ to 82 are displayed without and with a cubic isospin term using $p(A)=p_{0}+p_{1} A+p_{2} A^{2}$. Results have also been obtained for other shell regions, and the simultaneous treatment of several shell regions has so far been tested for $Z \geqslant 50$ with promising results. Particularly striking are the improved extrapolations for the p-rich rare-earth nuclei and for the region " $\mathrm{NW"}$ " of ${ }^{208} \mathrm{~Pb}$. Extrapolations are very sensitive to the function $p(A)$ and to the procedure used to extract $p(A)\left[\right.$ ref. $\left.\left.^{18}\right)\right]$.

Information about the physical origin of the higher-order terms in isospin has been obtained by testing theoretical binding energies ${ }^{19}$ ) for the $1 \mathrm{~d} 2 \mathrm{~s}$ shell for cubic contributions. The theoretical energies use experimental binding and excitation energies as input. The isospin dependence of the ground state binding energies indeed requires a small cubic term. The origin of the higher-order effects therefore includes subshell mixing, departures from the seniority-coupling scheme, and core polarization. The connection between these effects and the observed phenomenological $A$-dependence of each shell region is not understood and requires further study.

It is intended to continue the study of the $A$-dependence of the cubic isospin term for all regions and subsequently construct a global expression for use with the inhomogeneous equation in all shell regions including $Z>N$ (except $N=Z=$ odd). This has to be done with great care because of the established extreme sensitivity of the extrapolated values to the strength of the cubic term. 


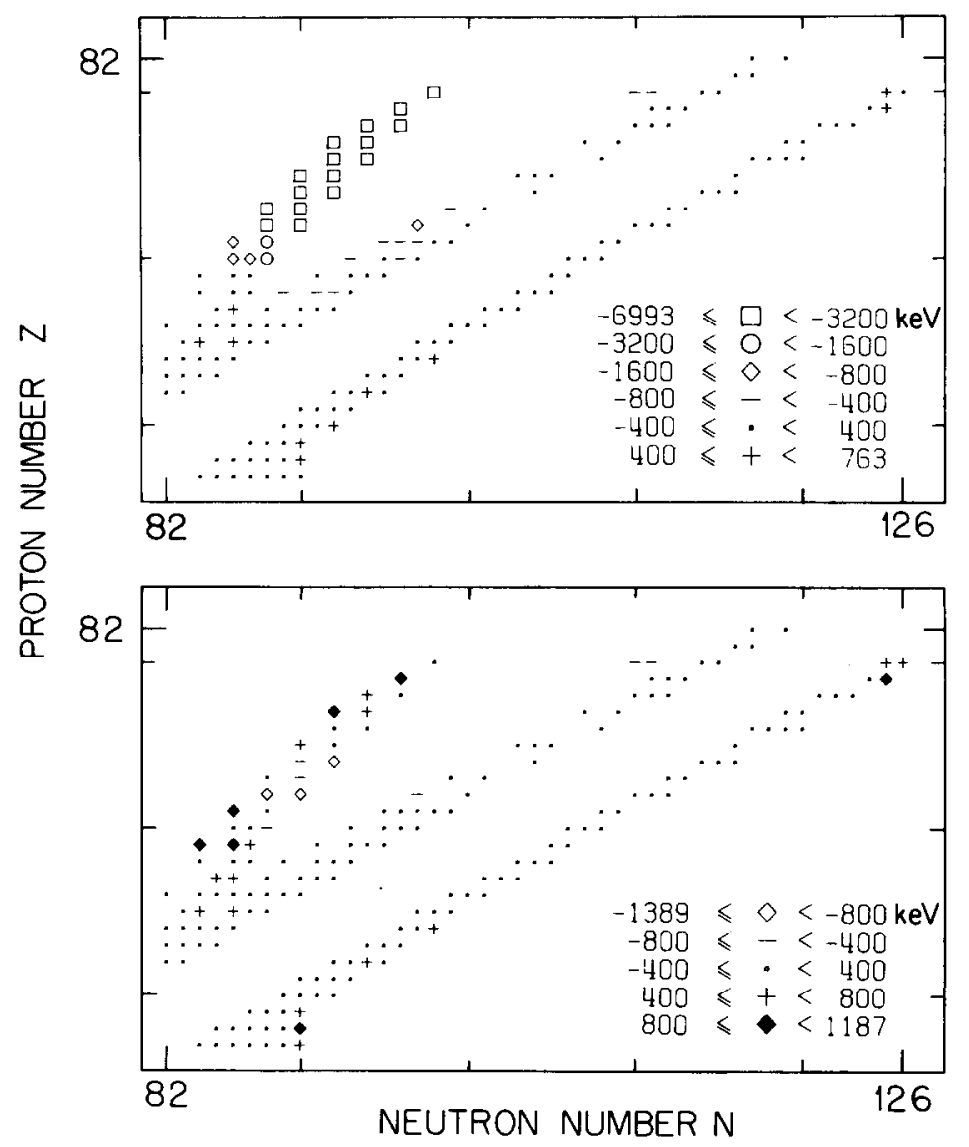

Fig. 11. Differences between experimental and calculated (extrapolated) masses for nuclei in the offdiagonal region $N=82$ to $126, Z=50$ to 82 from the solutions of an inhomogeneous third-order partial difference equation without (top) and with (bottom) a source term leading to a cubic dependence on isospin $T$ [see sect. 6; $p(A)=-15.6-0.01(A-170)+0.037(A-170)^{2} \mathrm{keV}$ ]. The empty band of nuclei of width $W=2 D=6$ along the line of $\beta$-stability. is used as data base.

\section{Conclusions}

Statistical tests provide only weak evidence for the existence of inhomogeneous terms in the transverse Garvey-Kelson relation and its associated mass equation. A systematic study of extrapolations using subsets of data consisting of bands of nuclei along the line of $\beta$-stability as data base shows pronounced effects in long-range extrapolations. The method is in principle applicable to any mass equation as a powerful test for the goodness of extrapolations. For the transverse Garvey-Kelson equation it is found that very ncutron-rich nuclei are on the average less stable than predicted, and very proton-rich nuclei are more stable. Shelldependent Coulomb energy and symmetry energy terms are recognized to contribute 
to an inhomogeneous source term but do not improve long-range extrapolations. Higher-order terms in isospin proportional to $T^{3}$ are found to have a profound influence on long-range extrapolations. Particularly subshell mixing and core polarization appear to be responsible for this effect which is strongly shell-dependent. Results for one shell region are presented.

Thanks are due to K.T. Hecht and I. Kelson for useful and stimulating discussion. The work was supported in part by the United States National Science Foundation and the United States-Israel Binational Science Foundation.

\section{References}

1) G.T. Garvey, W.J. Gerace, R.L. Jaffe, I. Talmi and I. Kelson, Rev. Mod. Phys. 41 (1969) S1

2) G.T. Garvey, Ann. Rev. Nucl. Sci. 19 (1969) 433

3) J. Jänecke, At. Nucl. Data Tables 17 (1976) 455

4) J. Jänecke and B.P. Eynon, At. Nucl. Data Tables 17 (1976) 467

5) J.E. Monahan and F.J.D. Serduke, Phys. Rev. C15 (1977) 1080

6) J.E. Monahan and F.J.D. Serduke, Phys. Rev. C17 (1978) 1196

7) J. Jänecke and H. Behrens, Phys. Rev. C9 (1973) 1276

8) J. Jänecke and B.P. Eynon, Nucl. Phys. A243 (1975) 326

9) J. Jänecke and H. Behrens, Z. Phys. 256 (1972) 236

10) E. Comay and I. Kelson, in Atomic masses and fundamental constants vol. 6, ed. J.A. Nolen and W. Benenson (Plenum, New York, 1979) p. 349

11) A.H. Wapstra, A 1981 midstream atomic mass evaluation, unpublished

12) E. Comay and I. Kelson, Z. Phys. A310 (1983) 107

13) E. Comay and I. Kelson, Phys. Rev. C29 (1984) 340

14) J. Jänecke, in Atomic masses and fundamental constants, vol 6, ed. J.A. Nolen and W. Benenson (Plenum, New York, 1979) p. 101

15) A. de-Shalit, Phys. Rev. 105 (1957) 1528

16) E. Comay and J. Jänecke, Nucl. Phys. A410 (1983) 103

17) J. Jänecke and E. Comay, Phys. Lett. 140B (1984) 1

18) J. Jänecke, E. Comay and P. Masson, unpublished

19) B.A. Brown, R. Radhi and B.H. Wildenthal, Phys. Reports 101 (1983) 315;

B.H. Wildenthal, private communication 